TRANSACTIONS OF THE

AMERICAN MATHEMATICAL SOCIETY

Volume 364, Number 12, December 2012, Pages 6495-6511

S 0002-9947(2012)05587-5

Article electronically published on June 8, 2012

\title{
SCALAR CURVATURE AND ASYMPTOTIC CHOW STABILITY OF PROJECTIVE BUNDLES AND BLOWUPS
}

\author{
ALBERTO DELLA VEDOVA AND FABIO ZUDDAS
}

\begin{abstract}
The holomorphic invariants introduced by Futaki as obstruction to asymptotic Chow semistability are studied by an algebraic-geometric point of view and are shown to be the Mumford weights of suitable line bundles on the Hilbert scheme of $\mathbb{P}^{n}$.

These invariants are calculated in two special cases. The first is a projective bundle $\mathbb{P}(E)$ over a curve of genus $g \geq 2$, and it is shown that it is asymptotically Chow polystable (with every polarization) if and only if the bundle $E$ is slope polystable. This proves a conjecture of Morrison with the extra assumption that the involved polarization is sufficiently divisible. Moreover it implies that $\mathbb{P}(E)$ is asymptotically Chow polystable (with every polarization) if and only if it admits a constant scalar curvature Kähler metric. The second case is a manifold blown up at points, and new examples of asymptotically Chow unstable constant scalar curvature Kähler classes are given.
\end{abstract}

\section{INTRODUCTION}

The existence on a compact complex manifold of a constant scalar curvature Kähler $(\operatorname{cscK})$ metric is a central problem in Kähler geometry and has been approached with a variety of geometric and analytical methods. While one can look for cscK metrics in arbitrary classes, major interest has been attracted by classes that are first Chern classes of ample line bundles. This is because of a conjecture of Yau 32, Tian [30, 31] and Donaldson [7] which relates the existence of a cscK metric in the first Chern class $c_{1}(A)$ of an ample line bundle $A$ on a manifold $M$, to the GIT stability of the polarized manifold $(M, A)$. More precisely the Yau-Tian-Donaldson conjecture states that a manifold $M$ admits a constant scalar curvature Kähler metric in the class $c_{1}(A)$ if and only if $(M, A)$ is K-polystable. The K-stability has been introduced by Tian [30] and then reformulated in a purely algebraic-geometric way by Donaldson [7]. While the "only if" part of the conjecture has been proved thanks to the works of Tian [30], Donaldson [7], Arezzo and Pacard 2, Stoppa 28, and Mabuchi [17, 19, the "if" part is widely open in general and has been proved only in the case of toric surfaces by Donaldson [8, and in the case of projective bundles over a curve thanks to the works of Narasimhan and Seshadri 22], and Ross and Thomas [25].

The K-stability is not the only GIT stability notion related to the existence of cscK metrics. Assuming that $M$ has no nontrivial holomorphic vector fields, Donaldson has proved that the existence of a $\operatorname{cscK}$ metric representing $c_{1}(A)$ implies the asymptotic Chow stability of $(M, A)$ 6]. Mabuchi has extended this result removing the hypothesis on the automorphisms and introducing a compatibility

Received by the editors November 15, 2010 and, in revised form, March 18, 2011.

2010 Mathematics Subject Classification. Primary 32Q15.

(C)2012 American Mathematical Society Reverts to public domain 28 years from publication 
hypothesis between the polarization $A$ and $\operatorname{Aut}(M)$ [16. Finally Futaki, Ono and Sano showed that Mabuchi's hypothesis is equivalent to the vanishing of certain holomorphic functionals, depending only on the polarization class $c_{1}(A)$, defined on the space of holomorphic vector fields with zeros of $M$ [11. These invariants have been previously introduced by Futaki as an obstruction to asymptotic Chow semistability [9]. Since they generalize the classical Futaki invariant, we will call them higher Futaki invariants.

As shown by Donaldson [7] and Paul and Tian 24, the original Futaki invariant has a purely algebraic-geometric nature and it is the Mumford weight of the socalled CM line $\lambda_{\mathrm{C} M}$ on the Hilbert scheme $\mathcal{H}$ of $\mathbb{P}^{N}$. We will show (section 2) that the same is true for the higher Futaki invariants.

Theorem 1.1. Let $\mathcal{H}$ be the Hilbert scheme of subschemes of $\mathbb{P}^{N}$ with Hilbert polynomial $\chi$. For every $\ell=1, \ldots, \operatorname{deg} \chi$ there are $\mathbb{Q}$-line bundles $\lambda_{C M, \ell}$ on $\mathcal{H}$ such that the higher Futaki invariant $F_{\ell}$ is the Mumford weight of $\lambda_{C M, \ell}$.

Furthermore we calculate the Chow weight and the higher Futaki invariants for two classes of manifolds: projective bundles over curves of genus at least two and blowups at finite sets of points. In the first case (section [3) we will show that the higher Futaki invariants are all proportional to the Futaki invariant. This, together with results of Narasimhan and Seshadri [22] and Mabuchi [16, is the main ingredient to prove the following.

Theorem 1.2. A projective bundle $\mathbb{P}(E)$ over a curve of genus $g \geq 2$ is asymptotically Chow polystable w.r.t. some (and hence any) polarization if and only if $E$ is slope polystable.

This proves a conjecture of Morrison with the extra assumption that the involved polarization is sufficiently divisible [20, Conjecture 5.10]. Actually Morrison's conjecture also deals with Hilbert stability, but we know that Hilbert stability and Chow stability asymptotically coincide thanks to a result of Mabuchi [18. We notice that one direction of a generalization of the Morrison conjecture to higherdimensional base manifolds has been recently proved by Seyyedali 27] using techniques different from ours. On the other hand a recent result due to Apostolov, Calderbank, Gauduchon and Tønnesen-Friedman [1] states that $\mathbb{P}(E)$ admits a cscK metric in some (and hence any) Kähler class if and only if $E$ is slope polystable. Thus we get the following.

Theorem 1.3. A projective bundle $\mathbb{P}(E)$ over a curve of genus $g \geq 2$ is cscK if and only if it is asymptotically Chow polystable w.r.t. some (and hence any) polarization.

In the case of blowups (section 4), we will give a formula for the Chow weight and higher Futaki invariants in terms of data on the base manifold. That formula, together with a result of Arezzo and Pacard [3], provides many new examples of asymptotically Chow unstable cscK classes. In particular we give the details in the case of $\mathbb{P}^{2}$ blown up at four points in special position.

Proposition 1.4. Let $M$ be the projective plane $\mathbb{P}^{2}$ blown up at four points. If all but one are aligned, then $M$ admits an asymptotically Chow unstable cscK polarization. 
Thus asymptotically Chow unstable cscK classes already exist in dimension two. To the best of our knowledge, the unique example of this kind previously known was the asymptotically Chow unstable Kähler-Einstein 7-dimensional toric manifold studied by Ono, Sano and Yotsutani [23. Finally it is worth noticing that, thanks to a theorem of Zhang [33, such manifolds admit no balanced embeddings (see 6] for a definition) for each sufficiently high power of the polarization. This is clear for the cases considered in Proposition 1.4. whose proof actually shows the instability of all sufficiently high powers of the polarization (see Example 4.3 for the details), but it is not a trivial consequence of the definition of Chow instability which, a priori, does not rule out the existence of an unbounded sequence of balanceable powers. However, to the best of the authors' knowledge, examples of such a phenomenon are not known.

\section{Higher CM Lines}

Let $(V, A)$ be an $n$-dimensional polarized variety or scheme. Given a oneparameter subgroup $\rho: \mathbb{C}^{*} \rightarrow \operatorname{Aut}(V)$ with a linearization on $A$, and denoted by $w(V, A)$, the weight of the $\mathbb{C}^{*}$-action induced on $\bigwedge^{\text {top }} H^{0}(V, A)$, we have the following definition, essentially due to Mumford [21]:

Definition 2.1. When $A$ is very ample and without higher cohomology, the (normalized) Chow weight of the given action is the rational number

$$
\operatorname{Chow}(V, A)=\frac{w(V, A)}{\chi(V, A)}-\frac{b_{0}(V, A)}{a_{0}(V, A)},
$$

where $a_{0}$ and $b_{0}$ are defined by $w\left(V, A^{k}\right)=b_{0}(V, A) k^{n+1}+O\left(k^{n}\right)$ and $\chi\left(V, A^{k}\right)=$ $a_{0}(V, A) k^{n}+O\left(k^{n-1}\right)$ as $k \gg 0$.

Clearly $w(V, A)$ and $b_{0}(V, A)$ depend on the fixed action $\rho$ and its linearization on $A$. On the other hand, Chow $(V, A)$ depends only on the action and it is insensible of the chosen linearization on $A$ (see Proposition 2.2 below). In the following we leave these dependences always understood since they will be clear from the context.

We are interested in the asymptotic behavior of $\operatorname{Chow}\left(V, A^{k}\right)$ when $k$ grows. By the general theory, at least for $k \gg 0$, we have the polynomial expansions

$$
\begin{aligned}
\chi\left(V, A^{k}\right) & =\sum_{\ell=0}^{n} a_{\ell}(V, A) k^{n-\ell}, \\
w\left(V, A^{k}\right) & =\sum_{\ell=0}^{n+1} b_{\ell}(V, A) k^{n+1-\ell},
\end{aligned}
$$

whence we get easily

$$
\begin{aligned}
& \operatorname{Chow}\left(V, A^{k}\right) \\
& =\frac{b_{n+1}(V, A)}{\chi\left(V, A^{k}\right)}+\frac{a_{0}(V, A)}{\chi\left(V, A^{k}\right)} \sum_{\ell=1}^{n} \frac{a_{0}(V, A) b_{\ell}(V, A)-b_{0}(V, A) a_{\ell}(V, A)}{a_{0}(V, A)^{2}} k^{n+1-\ell} .
\end{aligned}
$$

When $V$ is smooth, using equivariant cohomology theory we can express $b_{n+1}(V, A)$ by an integral of an equivariant characteristic class (see the proof of Proposition 2.2), and the localization formula gives $b_{n+1}(V, A)=0$ [10, Theorem 5.3.11]. In general the nonvanishing of $b_{n+1}$ turns out to be related to the nonreduced components of $V$. For example, let $V \subset \mathbb{P}^{2}$ be the thickened line cut out 
by the equation $x^{d}=0$. The $\mathbb{C}^{*}$-action defined by $\operatorname{diag}\left(t^{2}, t, t^{-3}\right)$ on $\mathbb{P}^{2}$ restricts to $V$ since $x^{d}$ is an invariant polynomial. Denote by $A$ the restriction to $V$ of the hyperplane bundle. Then $w\left(V, A^{k}\right)$ is nothing but the total weight of the induced action on the subspace of $\mathbb{C}[x, y, z] /\left(x^{d}\right)$ constituted by degree $k$ polynomials. Thus by trivial calculations we get

$$
w\left(V, A^{k}\right)=d k^{2}-d(2 d-3) k+d(d-1)(d-2)
$$

for all $k \geq d$, whence $b_{2}(V, A) \neq 0$ if $d>2$.

Proposition 2.2. In the situation above, for $\ell=1, \ldots, n$, let

$$
F_{\ell}(V, A)=\frac{a_{0}(V, A) b_{\ell}(V, A)-b_{0}(V, A) a_{\ell}(V, A)}{a_{0}(V, A)^{2}} .
$$

$F_{\ell}(V, A)$ does not depend on the linearization on $A$; moreover, if $V$ is smooth, then $F_{\ell}(V, A)$ is the $\ell$-th holomorphic invariant discussed in [11].

Proof. Changing the linearization of the given $\mathbb{C}^{*}$-action on $A$ has the effect of shifting the weight $w\left(V, A^{k}\right)$ to $w\left(V, A^{k}\right)+c k \chi\left(V, A^{k}\right)$ for some integral constant $c$. Thus $b_{\ell}(V, A)$ shifts to $b_{\ell}(V, A)+c a_{\ell}(V, A)$, and it is easy to see that $F_{\ell}(V, A)$ is unchanged.

To prove the second assertion we assume that $V$ is smooth and we use the (equivariant) Hirzebruch-Riemann-Roch theorem in the same way as Donaldson proves that $F_{1}$ is nothing but the Futaki invariant up to a constant [7. Let $\omega$ be an $S^{1}$-invariant Kähler form representing $c_{1}(A)$ and fix a moment map $\phi$, so that $\omega+\phi$ represents the equivariant first Chern class of $A$ in the Cartan model of the equivariant cohomology [4, 5]. Moreover let $\Theta$ be the curvature of the Chern connection $\nabla$ of the Kähler metric associated to $\omega$, and let $\Lambda=\nabla_{X}-L_{X}$, where $X$ is the holomorphic generator of the given $\mathbb{C}^{*}$-action and $L_{X}$ denotes the Lie derivative. The operator $\Lambda$ defines an endomorphism of the holomorphic tangent bundle and $\Theta+\Lambda$ represents the equivariant curvature of the fixed Kähler metric.

By the equivariant Hirzebruch-Riemann-Roch theorem [5, Theorem 8.2] we know that the character of the induced representation on $\bigoplus_{i \geq 0} H^{i}\left(V, A^{k}\right)^{(-1)^{i}}$ (here by the power -1 we mean the dual) is given by the integral of a suitable equivariant characteristic form. On the other hand, by ampleness of $A$ we have $H^{i}\left(V, A^{k}\right)=0$ for all $i>0$ as $k \gg 0$. Thus we get

$$
\begin{aligned}
w\left(V, A^{k}\right) & =\left.\frac{d}{d t}\right|_{t=0} \int_{V} e^{k(\omega+t \phi)} \operatorname{td}(\Theta+t \Lambda) \\
& =\int_{V} k \phi e^{k \omega} \operatorname{td}(\Theta)+\left.\int_{V} e^{k \omega} \frac{d}{d t}\right|_{t=0} \operatorname{td}(\Theta+t \Lambda) \\
& =\sum_{\ell=0}^{n} k^{n+1-\ell} \int_{V} \phi \frac{\omega^{n-\ell}}{(n-\ell) !} \operatorname{td}_{\ell}(\Theta)+\sum_{\ell=0}^{n} k^{n-\ell} \int_{V} \frac{\omega^{n-\ell}}{(n-\ell) !} \operatorname{td}_{\ell+1}(\Theta+\Lambda) \\
& =\sum_{\ell=0}^{n} k^{n+1-\ell}\left(\int_{V} \phi \frac{\omega^{n-\ell}}{(n-\ell) !} \operatorname{td}_{\ell}(\Theta)+\int_{V} \frac{\omega^{n+1-\ell}}{(n+1-\ell) !} \operatorname{td}_{\ell}(\Theta+\Lambda)\right)
\end{aligned}
$$

and

$$
\chi\left(V, A^{k}\right)=\int_{V} e^{k \omega} \operatorname{td}(\Theta)=\sum_{\ell=0}^{n} k^{n-\ell} \int_{V} \frac{\omega^{n-\ell}}{(n-\ell) !} \operatorname{td}_{\ell}(\Theta) .
$$


Letting $\hat{\phi}=b_{0}(V, A) / a_{0}(V, A)=\left(\int_{V} \frac{\omega^{n}}{n !}\right)^{-1} \int_{V} \phi \frac{\omega^{n}}{n !}$ be the average of $\phi$, we have

$$
F_{\ell}(V, A)=\frac{\int_{V}(\phi-\hat{\phi}) \frac{\omega^{n-\ell}}{(n-\ell) !} \operatorname{td}_{\ell}(\Theta)}{\int_{V} \frac{\omega^{n}}{n !}}+\frac{\int_{V} \frac{\omega^{n+1-\ell}}{(n+1-\ell) !} \operatorname{td}_{\ell}(\Theta+\Lambda)}{\int_{V} \frac{\omega^{n}}{n !}},
$$

which are essentially the coefficients of the polynomial expansion (4) of 11] if we assume $\hat{\phi}=0$.

We can use the Chow-Mumford weight introduced above and test configurations [7. 26] to define the (asymptotic) Chow stability.

Definition 2.3. A test configuration $(X, L) \rightarrow \mathbb{C}$ of exponent $k>0$ of a polarized manifold $(M, A)$ consists of a scheme $X$ endowed with a $\mathbb{C}^{*}$-action that linearizes on a line bundle $L$ over $X$, and a flat $\mathbb{C}^{*}$-equivariant morphism $f: X \rightarrow \mathbb{C}$ (where $\mathbb{C}^{*}$ acts on $\mathbb{C}$ by multiplication) such that $\left.L\right|_{f^{-1}(0)}$ is ample on $f^{-1}(0)$ and we have $\left(f^{-1}(1),\left.L\right|_{f^{-1}(1)}\right) \simeq\left(M, A^{k}\right)$.

When $(M, A)$ has a $\mathbb{C}^{*}$-action $\rho: \mathbb{C}^{*} \rightarrow \operatorname{Aut}(M)$, a test configuration where $X=M \times \mathbb{C}$ and $\mathbb{C}^{*}$ acts on $X$ diagonally through $\rho$ is called product configuration. A product configuration endowed with the trivial $\mathbb{C}^{*}$-action on $M$ is called a trivial configuration.

Clearly the central fiber $\left(f^{-1}(0),\left.L\right|_{f^{-1}(0)}\right)$ of a test configuration is a polarized scheme endowed with a $\mathbb{C}^{*}$-action.

Definition 2.4. The polarized manifold $(M, A)$ is called

- asymptotically Chow semistable if there is a $k_{0}>0$ such that for each test configuration of exponent $k>k_{0}$ the Chow weight of the induced action on the central fiber $\left(f^{-1}(0),\left.L\right|_{f^{-1}(0)}\right)$ is less than or equal to zero;

- asymptotically Chow polystable if it is semistable and the Chow weight is zero if and only if we have a product configuration;

- asymptotically Chow stable if it is polystable and every product configuration is trivial;

- asymptotically Chow unstable if it is not semistable.

With the notation introduced above, the main theorem of [16] becomes

Theorem 2.5 (Mabuchi). Let $(M, A)$ be a polarized $n$-fold admitting a cscK metric representing $c_{1}(A)$. Then $(M, A)$ is asymptotically Chow polystable if and only if for every $\mathbb{C}^{*}$-action linearizing on $A$ we have $F_{\ell}(M, A)=0$ for all $\ell=1, \ldots, n$.

Paul and Tian 24] have shown that the Futaki invariant is the Mumford weight (in the sense of GIT) of the line bundle $\lambda_{\mathrm{C} M}$ over the Hilbert scheme $\mathcal{H}$ of the subschemes of $\mathbb{P}^{N}$ with Hilbert polynomial

$$
\chi(k)=a_{0} k^{n}+a_{1} k^{n-1}+\cdots+a_{n} .
$$

In the same way the invariant $F_{\ell}$ is the Mumford weight of the line bundle $\lambda_{\mathrm{C} M, \ell}$, which is constructed as follows.

Let $f: \mathcal{U} \rightarrow \mathcal{H}$ be the universal (flat) family over $\mathcal{H}$. By general theory there is an embedding $\iota: \mathcal{U} \rightarrow \mathcal{H} \times \mathbb{P}^{N}$ and $f$ factorizes through it: $f=p_{r_{\mathcal{H}}} \circ \iota$. Moreover let $\mathcal{L}=\iota^{*} \circ p r_{\mathbb{P}^{N}}^{*} \mathcal{O}_{\mathbb{P}^{N}}(1)$ be the relative very ample line bundle on $\mathcal{U}$ obtained by restriction of the hyperplane bundle on each fiber of $f$.

Thanks to the relative very ampleness of $\mathcal{L}$, the cohomology $H^{0}\left(\mathcal{U}_{x}, \mathcal{L}_{x}^{k}\right)$ of the fiber of $f$ over $x \in \mathcal{H}$ is isomorphic to the fiber over the same point of the direct 
image $f_{*}\left(\mathcal{L}^{k}\right)$, at least for $k$ sufficiently large. Indeed, with this assumption, by the flatness hypothesis the direct image $f_{*}\left(\mathcal{L}^{k}\right)$ is a locally free sheaf on $\mathcal{H}[12$, Proposition 7.9.13], and by relative ampleness we have $H^{q}\left(\mathcal{U}_{x}, \mathcal{L}_{x}^{k}\right)=0$ for all $q>0$ so that the fiber $f_{*}\left(\mathcal{L}^{k}\right)_{x}$ is naturally isomorphic to $H^{0}\left(\mathcal{U}_{x}, \mathcal{L}_{x}^{k}\right)$ by [13, Theorem 12.11] as claimed. Thus we conclude that

$$
\begin{aligned}
\operatorname{rk} f_{*}\left(\mathcal{L}^{k}\right) & =\operatorname{dim} H^{0}\left(\mathcal{U}_{x}, \mathcal{L}_{x}^{k}\right), \\
\left.\operatorname{det} f_{*}\left(\mathcal{L}^{k}\right)\right|_{x} & =\operatorname{det} H^{0}\left(\mathcal{U}_{x}, \mathcal{L}_{x}^{k}\right),
\end{aligned}
$$

for all $x \in \mathcal{H}$ and $k \gg 0$. Since $\operatorname{dim} H^{0}\left(\mathcal{U}_{x}, \mathcal{L}_{x}^{k}\right)$ coincides with the Hilbert polynomial $\chi(k)$, we have an expansion

$$
\operatorname{rk} f_{*}\left(\mathcal{L}^{k}\right)=a_{0} k^{n}+a_{1} k^{n-1}+\cdots+a_{n} \quad \text { for } k \gg 0 .
$$

Now consider the determinant of the locally free sheaf $f_{*}\left(\mathcal{L}^{k}\right)$ for $k$ big enough. As an easy corollary of a result due to Knudsen and Mumford [14, Proposition 4], we have

$$
\operatorname{det} f_{*}\left(\mathcal{L}^{k}\right)=\mu_{0}^{k^{n+1}} \otimes \mu_{1}^{k^{n}} \otimes \cdots \otimes \mu_{n+1},
$$

where $\mu_{0}, \ldots, \mu_{n+1}$ are $\mathbb{Q}$-line bundles on $\mathcal{H}$.

Definition 2.6. The Chow-line on the Hilbert scheme $\mathcal{H}$ is defined by

$$
\lambda_{\text {Chow }}(\mathcal{H}, \mathcal{L})=\operatorname{det} f_{*}(\mathcal{L})^{\frac{1}{r \mathrm{k} f_{*}(\mathcal{L})}} \otimes \mu_{0}^{-\frac{1}{a_{0}}} .
$$

Combining (77) and (6), always for $k \gg 0$, we get the asymptotic expansion

$$
\begin{aligned}
\lambda_{\text {Chow }}\left(\mathcal{H}, \mathcal{L}^{k}\right) & =\left(\operatorname{det} f_{*}\left(L^{k}\right)^{\frac{1}{a_{0}}} \otimes \mu_{0}^{-\frac{k \chi(k)}{a_{0}^{2}}}\right)^{\frac{a_{0}}{\chi(k)}} \\
& =\mu_{n+1}^{\frac{1}{\chi(k)}} \otimes \bigotimes_{\ell=1}^{n}\left(\mu_{\ell}^{\frac{1}{a_{0}}} \otimes \mu_{0}^{-\frac{a_{\ell}}{a_{0}^{2}}}\right)^{\frac{a_{0} k^{n+1-\ell}}{\chi(k)}} .
\end{aligned}
$$

Definition 2.7. The $\ell$-th CM-line on the Hilbert scheme $\mathcal{H}$ is defined by

$$
\lambda_{\mathrm{C} M, \ell}(\mathcal{H}, \mathcal{L})=\mu_{\ell}^{\frac{1}{a_{0}}} \otimes \mu_{0}^{-\frac{a_{\ell}}{a_{0}^{2}}} .
$$

For $\ell=1$ we recover (a rational multiple of) the CM-line introduced by Paul and Tian 24 .

Since $S L(N+1, \mathbb{C})$ acts naturally on $\mathcal{H}$ and $\mathcal{U}$ making equivariant all maps introduced above, $\mathcal{L}$ comes equipped with a natural $S L(N+1, \mathbb{C})$-linearization and expansion (7) holds in the sense of linearized bundles. Hence the $\lambda_{\mathrm{C} M, \ell}$ are linearized bundles. Fix a one-parameter subgroup of $S L(N+1, \mathbb{C})$, and let $x \in \mathcal{H}$ be a fixed point; moreover set $V=\mathcal{U}_{x}$ and $A=\mathcal{L}_{x}$. Thus we are in the situation of Definition 2.1 and are defining $b_{\ell}(V, A)$ as in (2). On the other hand by (5) the Mumford weight of $\left.\operatorname{det} f_{*}\left(\mathcal{L}^{k}\right)\right|_{x}$ is nothing but $w\left(V, A^{k}\right)$; thus by (77) the Mumford weight of $\mu_{\ell}$ over $x$ must be $b_{\ell}$. This proves the following.

Proposition 2.8. The invariants Chow and $F_{\ell}$ are the Mumford weights of the lines $\lambda_{C h o w}$ and $\lambda_{C M, \ell}$ respectively. 


\section{Projective Bundles over Curves}

Let $\Sigma$ be a genus $g \geq 2$ smooth curve and let $E$ be a rank $n \geq 2$ vector bundle on $\Sigma$. Let $M=\mathbb{P}(E)$ be the projective bundle associated to $E$ and denote by $\pi: M \rightarrow$ $\Sigma$ the projection. Each line bundle on $\mathbb{P}(E)$ has the form $L=\mathcal{O}_{\mathbb{P}(E)}(r) \otimes \pi^{*} B$, where $\mathcal{O}_{\mathbb{P}(E)}(1)$ is the fiberwise hyperplane bundle, $B$ is a line bundle on $\Sigma$, and $r \in \mathbb{Z}$. Assume that $r$ and $B$ are such that $L$ is ample. The pair $(M, L)$ turns out to be an $n$-dimensional polarized manifold, and if $E=E_{1} \oplus \cdots \oplus E_{s}$ is the (unique up to the order) decomposition in indecomposable vector bundles, then every $\mathbb{C}^{*}$-action on $\mathbb{P}(E)$ is induced by a fiberwise action on $E$ of the form

$$
t \cdot\left(e_{1}, \ldots, e_{s}\right)=\left(t^{\lambda_{1}} e_{1}, \ldots, t^{\lambda_{s}} e_{s}\right),
$$

with $\lambda_{j} \in \mathbb{Z}$. Finally let $\lambda_{0}$ be the weight of a fiberwise $\mathbb{C}^{*}$-action on $B$.

Proposition 3.1. In the situation above we have

$$
\begin{array}{r}
\operatorname{Chow}\left(M, L^{k}\right)=\frac{\chi\left(\Sigma, \operatorname{det}\left(E \otimes B^{-\frac{1}{r}}\right)\right)}{\mu\left(E \otimes B^{-\frac{1}{r}}\right) \chi\left(\Sigma, S^{k r}\left(E^{*} \otimes B^{\frac{1}{r}}\right)\right)} \\
\times \frac{\left(\begin{array}{c}
n-1+k r \\
n
\end{array}\right)}{n+1} \sum_{j=1}^{s} \lambda_{j} \operatorname{rk}\left(E_{j}\right)\left(\mu\left(E_{j}\right)-\mu(E)\right),
\end{array}
$$

where $\mu(F)=\operatorname{deg}(F) / \operatorname{rk}(F)$ is the slope of the bundle $F$.

Remark 3.2. Although $E \otimes B^{-\frac{1}{r}}$ might not be a genuine vector bundle on $\Sigma$, its Chern classes are well defined as well as the formula above.

Proof. By Definition 2.1 of Chow weight we need to calculate $\chi\left(M, L^{k}\right)$ and $w\left(M, L^{k}\right)$. By the projection formula we have $\pi_{*}\left(L^{k}\right) \simeq S^{k r} E^{*} \otimes B^{k}$ equivariantly; thus $\chi\left(M, L^{k}\right)=\chi\left(\Sigma, S^{k r} E^{*} \otimes B^{k}\right)$ and $w\left(M, L^{k}\right)=w\left(\Sigma, S^{k r} E^{*} \otimes B^{k}\right)$.

Thus, by the Hirzebruch-Riemann-Roch theorem and equalities

$$
\operatorname{rk}\left(S^{k r} E^{*}\right)=\left(\begin{array}{c}
n-1+k r \\
k r
\end{array}\right) \quad \text { and } \quad c_{1}\left(S^{k r} E^{*}\right)=-\left(\begin{array}{c}
n-1+k r \\
k r-1
\end{array}\right) c_{1}(E)
$$

we have

$$
\begin{aligned}
\chi\left(M, L^{k}\right) & =\int_{\Sigma} \operatorname{ch}\left(S^{k r} E^{*}\right) \operatorname{ch}\left(B^{k}\right) \operatorname{td}(\Sigma) \\
& =\int_{\Sigma} c_{1}\left(S^{k r} E^{*}\right)+\operatorname{rk}\left(S^{k r} E^{*}\right) \int_{\Sigma} c_{1}\left(B^{k}\right)+\operatorname{rk}\left(S^{k r} E^{*}\right) \int_{\Sigma} \frac{c_{1}(\Sigma)}{2} \\
& =-\left(\begin{array}{c}
n-1+k r \\
k r-1
\end{array}\right) \int_{\Sigma} c_{1}(E)+\left(\begin{array}{c}
n-1+k r \\
k r
\end{array}\right)\left(k \int_{\Sigma} c_{1}(B)+\int_{\Sigma} \frac{c_{1}(\Sigma)}{2}\right) \\
& =\left(\begin{array}{c}
n-1+k r \\
k r
\end{array}\right)(1-g-k r \mu(E)+k \operatorname{deg}(B)) .
\end{aligned}
$$

To calculate $w\left(M, L^{k}\right)$, which is equal to $w\left(\Sigma, S^{k r} E^{*} \otimes B^{k}\right)$ as noticed above, we use the Equivariant Hirzebruch-Riemann-Roch theorem. We have to integrate the degree $(2,2)$ component of the equivariant cohomology class $\operatorname{ch}\left(S^{k r} E^{*}\right) \operatorname{ch}\left(B^{k}\right) \operatorname{td}(\Sigma)$ (here we are using the same notation as in the nonequivariant case since we deal only with equivariant classes until the end of the proof). Since the given fiberwise actions on $E$ and $B$ cover the trivial action on $\Sigma$, the equivariant class $\operatorname{td}(\Sigma)=1+\frac{c_{1}(\Sigma)}{2}$ 
has no degree $(2,2)$ part. On the other hand, by tedious but elementary calculation we get

$$
\operatorname{ch}_{2}\left(S^{k r} E^{*}\right)=\left(\begin{array}{c}
n+k r \\
k r-1
\end{array}\right) \frac{c_{1}(E)^{2}-2 c_{2}(E)}{2}+\left(\begin{array}{c}
n-1+k r \\
k r-2
\end{array}\right) \frac{c_{1}(E)^{2}}{2}
$$

hence

$$
\begin{aligned}
w\left(M, L^{k}\right)= & \int_{\Sigma} \operatorname{ch}_{2}\left(S^{k r} E^{*}\right)+\int_{\Sigma} c_{1}\left(S^{k r} E^{*}\right) \frac{c_{1}(\Sigma)}{2}+\int_{\Sigma} c_{1}\left(S^{k r} E^{*}\right) c_{1}\left(B^{k}\right) \\
& +\operatorname{rk}\left(S^{k r} E^{*}\right) \int_{\Sigma} c_{1}\left(B^{k}\right) \frac{c_{1}(\Sigma)}{2}+\operatorname{rk}\left(S^{k r} E^{*}\right) \int_{\Sigma} \frac{c_{1}\left(B^{k}\right)^{2}}{2} \\
= & \left(\begin{array}{c}
n+k r \\
k r-1
\end{array}\right) \int_{\Sigma} \frac{c_{1}(E)^{2}-2 c_{2}(E)}{2}+\left(\begin{array}{c}
n-1+k r \\
k r-2
\end{array}\right) \int_{\Sigma} \frac{c_{1}(E)^{2}}{2} \\
& -\left(\begin{array}{c}
n-1+k r \\
k r-1
\end{array}\right) \int_{\Sigma} \frac{c_{1}(E) c_{1}(\Sigma)}{2}-\left(\begin{array}{c}
n-1+k r \\
k r-1
\end{array}\right) k \int_{\Sigma} c_{1}(E) c_{1}(B) \\
& +\left(\begin{array}{c}
n-1+k r \\
k r
\end{array}\right) k \int_{\Sigma} \frac{c_{1}(B) c_{1}(\Sigma)}{2}+\left(\begin{array}{c}
n-1+k r \\
k r
\end{array}\right) k^{2} \int_{\Sigma} \frac{c_{1}(B)^{2}}{2}
\end{aligned}
$$

We will use the Cartan model of equivariant cohomology to calculate the equivariant characteristic numbers above. To this end, fix an $S^{1}$-invariant Hermitian metric on each $E_{j}$ of the decomposition $E=E_{1} \oplus \cdots \oplus E_{s}$. The sum of such metrics gives a Hermitian metric on $E$, whose equivariant curvature of the compatible connection has the form $\Theta+\Lambda$, where $\Theta=\Theta_{1} \oplus \cdots \oplus \Theta_{s}$ is the direct sum of nonequivariant curvatures of the fixed metrics on the $E_{j}$ 's, and $\Lambda$ is the endomorphism of $E$ represented by $\lambda_{1} I_{1} \oplus \cdots \oplus \lambda_{s} I_{s}, I_{j}$ being the identity on $E_{j}$. By equivariant Chern-Weil theory we have $c_{1}(E)=[\operatorname{tr}(\Theta)+\operatorname{tr}(\Lambda)]$ and $c_{1}(E)^{2}-2 c_{2}(E)=\left[2 \operatorname{tr}(\Theta \Lambda)+\operatorname{tr}\left(\Lambda^{2}\right)\right]$, and by construction $\operatorname{tr}(\Lambda)=\sum_{j=1}^{s} \lambda_{j} \operatorname{rk}\left(E_{j}\right)$ and $\operatorname{tr}(\Theta \Lambda)=\sum_{j=1}^{s} \lambda_{j} \operatorname{tr}\left(\Theta_{j}\right)$. In the same way, if we fix a Hermitian metric on $B$ with curvature $\omega$, we have $c_{1}(B)=\left[\omega+\lambda_{0}\right]$. We calculate

$$
\begin{aligned}
w\left(M, L^{k}\right)= & \left(\begin{array}{c}
n+k r \\
k r-1
\end{array}\right) \int_{\Sigma} \operatorname{tr}(\Theta \Lambda)+\left(\begin{array}{c}
n-1+k r \\
k r-2
\end{array}\right) \operatorname{tr}(\Lambda) \int_{\Sigma} \operatorname{tr}(\Theta) \\
& -\left(\begin{array}{c}
n-1+k r \\
k r-1
\end{array}\right)\left[\operatorname{tr}(\Lambda) \int_{\Sigma} \frac{c_{1}(\Sigma)}{2}-k\left(\lambda_{0} \int_{\Sigma} \operatorname{tr}(\Theta)+\operatorname{tr}(\Lambda) \int_{\Sigma} \omega\right)\right] \\
& +\left(\begin{array}{c}
n-1+k r \\
k r
\end{array}\right)\left[k \lambda_{0} \int_{\Sigma} \frac{c_{1}(\Sigma)}{2}+k^{2} \lambda_{0} \int_{\Sigma} \omega\right] \\
= & \left(\begin{array}{c}
n-1+k r \\
k r
\end{array}\right)\left[\frac{k r(n+k r)}{n(n+1)} \int_{\Sigma} \operatorname{tr}(\Theta \Lambda)+\frac{k r(k r-1)}{n+1} \operatorname{tr}(\Lambda) \mu(E)\right. \\
& -\frac{k r}{n} \operatorname{tr}(\Lambda)(1-g)-\frac{k^{2} r}{n} \operatorname{tr}(\Lambda) \int_{\Sigma} \omega-\frac{k^{2} r}{n} \lambda_{0} \int_{\Sigma} \operatorname{tr}(\Theta) \\
& \left.+k \lambda_{0}(1-g)+k^{2} \lambda_{0} \int_{\Sigma} \omega\right] \\
= & \left(\begin{array}{c}
n-1+k r \\
k r
\end{array}\right)\left[\frac{k r(n+k r)}{n(n+1)} \sum_{j=1}^{s} \lambda_{j} \operatorname{rk}\left(E_{j}\right)\left(\mu\left(E_{j}\right)-\mu(E)\right)\right. \\
& \left.+k\left(\lambda_{0}-\frac{r}{n} \operatorname{tr}(\Lambda)\right)(1-g-k r \mu(E)+k \operatorname{deg}(B))\right] .
\end{aligned}
$$


By Definition 2.1, subtracting from the ratio $w\left(M, L^{k}\right) / \chi\left(M, L^{k}\right)$ the linear term in $k$, we get

$$
\begin{aligned}
\operatorname{Chow}\left(M, L^{k}\right)=\frac{1-g+\operatorname{deg}(E)-\frac{n}{r} \operatorname{deg}(B)}{\left(\mu(E)-\frac{1}{r} \operatorname{deg}(B)\right)(1-g-k r \mu(E)+k \operatorname{deg}(B))} \\
\quad \times \frac{k r}{n(n+1)} \sum_{j=1}^{s} \lambda_{j} \operatorname{rk}\left(E_{j}\right)\left(\mu\left(E_{j}\right)-\mu(E)\right),
\end{aligned}
$$

and the thesis follows.

At this point we recall the following.

Definition 3.3. A vector bundle $E$ over a smooth curve $\Sigma$ is called

- slope semistable if $\mu(F) \leq \mu(E)$ for every subbundle $F \subset E$,

- slope stable if $\mu(F)<\mu(E)$ for every subbundle $F \subset E$,

- slope polystable if $E$ decomposes as $E=E_{1} \oplus \cdots \oplus E_{s}$, where the $E_{j}$ 's are stable vector bundles satisfying $\mu\left(E_{j}\right)=\mu(E)$.

We also recall that for every line bundle $B$ on $\Sigma$ we have $\mu(E \otimes B)=\mu(E)+$ $\operatorname{deg}(B)$; thus $E$ is slope (semi/poly)stable if and only if $E \otimes B$ is.

Thanks to Proposition 3.1 the invariants $F_{\ell}$ defined in the statement of Proposition 2.2 can be easily calculated in the considered case.

Corollary 3.4. In the situation above we have $\operatorname{Chow}\left(M, L^{k}\right)=0$ if and only if $F_{\ell}(M, L)=0$ for some (and hence any) $\ell=1, \ldots, n$. More precisely there are rational constants $C_{\ell}>0$, depending only on $n$, such that

$$
F_{\ell}(M, L)=-C_{\ell} \frac{\chi\left(\Sigma, \operatorname{det}\left(E \otimes B^{-\frac{1}{r}}\right)\right)}{\mu\left(E \otimes B^{-\frac{1}{r}}\right)^{2}} \sum_{j=1}^{s} \lambda_{j} \operatorname{rk}\left(E_{j}\right)\left(\mu\left(E_{j}\right)-\mu(E)\right)
$$

In particular $F_{\ell}(M, L)=0$ for all $\mathbb{C}^{*}$-actions if and only if $\mu\left(E_{j}\right)=\mu(E)$ for all $j=1, \ldots, s$.

Proof. By definition, $F_{\ell}(M, L)$ is the coefficient of $k^{n+1-\ell}$ in the polynomial $\frac{\chi\left(M, L^{k}\right)}{a_{0}(M, L)} \operatorname{Chow}\left(M, L^{k}\right)$. Thanks to Proposition 3.1 (and its proof) we have $a_{0}(M, L)=-\frac{\mu\left(E \otimes B^{-\frac{1}{r}}\right)}{(n-1) !}$ and

$$
\begin{aligned}
& \frac{\chi\left(M, L^{k}\right)}{a_{0}(M, L)} \operatorname{Chow}\left(M, L^{k}\right) \\
& \quad=-\frac{(n-1) !}{n+1}\left(\begin{array}{c}
n-1+k \\
n
\end{array}\right) \frac{\chi\left(\Sigma, \operatorname{det} E \otimes B^{-\frac{1}{r}}\right)}{\mu\left(E \otimes B^{-\frac{1}{r}}\right)^{2}} \sum_{j=1}^{s} \lambda_{j} \operatorname{rk}\left(E_{j}\right)\left(\mu\left(E_{j}\right)-\mu(E)\right) .
\end{aligned}
$$

Thus (9) is proved with constants $C_{\ell}$ generated by

$$
\sum_{\ell=1}^{n} C_{\ell} k^{n+1-\ell}=\frac{1}{n(n+1)} \prod_{i=0}^{n-1}(k+i)
$$


To prove the last assertion it remains to show that $\chi\left(\Sigma, \operatorname{det}\left(E \otimes B^{-\frac{1}{r}}\right)\right)$ is nonzero. Ampleness of $L$ implies $\chi\left(M, L^{k}\right)>0$ for $k \gg 0$, but on the other hand, $\chi\left(M, L^{k}\right)=$ $-\frac{\operatorname{deg}\left(E \otimes B^{-\frac{1}{r}}\right)}{n !} k^{n}+O\left(k^{n-1}\right)$; thus we get $\operatorname{deg}\left(E \otimes B^{-\frac{1}{r}}\right)<0$. By that and $g \geq 2$ we obtain

$$
\chi\left(\Sigma, \operatorname{det}\left(E \otimes B^{-\frac{1}{r}}\right)\right)=\operatorname{deg}\left(E \otimes B^{-\frac{1}{r}}\right)+1-g<0,
$$

and we are done.

The relation between slope stability of $E$ and Chow (and Hilbert) stability of $\mathbb{P}(E)$ has been initially investigated by Morrison [20] when $\operatorname{rk}(E)=2$. He conjectured that the Chow and Hilbert stability of $\mathbb{P}(E)$ are equivalent to slope stability of $E$ [20, Conjecture 5.10]. Combining the theorems above and the results of Mabuchi [16], we get the following result, which proves the Morrison conjecture for $E$ of every rank, with the extra hypothesis that the chosen polarization on $\mathbb{P}(E)$ is sufficiently divisible.

Theorem 3.5. A projective bundle $\mathbb{P}(E)$ over a curve of genus $g \geq 2$ is asymptotically Chow (poly)stable with respect to some (and hence any) polarization if and only if $E$ is slope (poly) stable.

Proof. Assume that $E$ is slope polystable. In this case from a well-known result of Narasimhan and Seshadri 22 it follows that $\mathbb{P}(E)$ admits a cscK metric in each Kähler class. Moreover, by Corollary [3.4, all the invariants $F_{\ell}$ are zero. Thus by Mabuchi's Theorem 2.5] $\mathbb{P}(E)$ is asymptotically Chow polystable. In particular, if $E$ is stable, then it is indecomposable and $\mathbb{P}(E)$ admits no nontrivial $\mathbb{C}^{*}$-actions and it is stable.

Before proving the other direction we notice that Ross and Thomas proved that if $E$ is unstable, then $\mathbb{P}(E)$ is $K$-unstable [25. Theorem 5.13]; hence it is also asymptotically Chow unstable. On the other hand, restricting to the semistable case does not simplify sensibly the argument below. For this reason and for the sake of completeness we treat here the general case.

Assume that $E$ is not slope polystable. This means that we have a subbundle $F \subset E$ such that either $\mu(F)>\mu(E)$ and $E=F \oplus E / F$, or $\mu(F) \geq \mu(E)$ and $F$ is not a direct summand. In the first case, $\mathbb{P}(E)$ is not asymptotically Chow polystable since, taking the $\mathbb{C}^{*}$-action defined by $\operatorname{diag}(t, 1)$, the (asymptotic) Chow weight is nonzero thanks to Proposition 3.1. In the second case, since $F$ is not a direct summand of $E$, the exact sequence

$$
0 \rightarrow F \rightarrow E \rightarrow E / F \rightarrow 0
$$

gives a nonzero element $e \in H^{1}\left(\Sigma,(E / F)^{*} \otimes F\right)$ by which one can construct a nonproduct test configuration for $\mathbb{P}(E)$ with central fiber isomorphic to $\mathbb{P}(F \oplus E / F)$ and nonnegative Chow weight (compare with Ross and Thomas [25, Remark 5.14]). We need to make this construction explicit to find the induced action on the central fiber and then compute the Chow weight. To this end, let $\Sigma=\bigcup_{i} U_{i}$ be a cover by holomorphic discs. On each $U_{i}$ fix a trivialization of $F$ and $E / F$ with transition functions $f_{i j}: U_{i} \cap U_{j} \rightarrow G L(\operatorname{rk}(F), \mathbb{C}), g_{i j}: U_{i} \cap U_{j} \rightarrow G L(\operatorname{rk}(E / F), \mathbb{C})$. Then we can trivialize $E$ over every $U_{i}$ such that the transition functions $e_{i j}: U_{i} \cap U_{j} \rightarrow$ $G L(\operatorname{rk}(E), \mathbb{C})$ are upper triangular; namely, they have the form

$$
e_{i j}=\left(\begin{array}{cc}
f_{i j} & h_{i j} \\
0 & g_{i j}
\end{array}\right)
$$


for some $h_{i j}: U_{i} \cap U_{j} \rightarrow \operatorname{Mat}(\operatorname{rk}(F) \times \operatorname{rk}(E / F), \mathbb{C})$. It is easy to check that $\left\{h_{i j}\right\}$ defines a Cech 1-cocycle of the sheaf of sections of $(E / F)^{*} \otimes F$ whose cohomology class $e$ determines the extension (10) up to isomorphism. For any $z \in \mathbb{C}$, let $E_{z}$ be the vector bundle on $\Sigma$ having transition functions given by

$$
\tilde{e}_{i j}=\left(\begin{array}{cc}
f_{i j} & z h_{i j} \\
0 & g_{i j}
\end{array}\right) .
$$

Clearly $E_{0} \simeq F \oplus E / F$. Moreover $\tilde{e}_{i j}=\operatorname{diag}(z, 1) e_{i j} \operatorname{diag}\left(z^{-1}, 1\right)$, whence $E_{z} \simeq E$ for all $z \neq 0$. Considering the $\tilde{e}_{i j}$ 's as transition functions relative to the open covering $\left\{U_{i} \times \mathbb{C}\right\}$ of $\Sigma \times \mathbb{C}$ gives a vector bundle $\mathcal{E} \rightarrow \Sigma \times \mathbb{C}$, whose restriction to $\Sigma \times\{z\}$ is nothing but $E_{z}$. At this point we claim that the $\mathbb{C}^{*}$-action defined by $t \cdot(x, z)=(x, t z)$ on $\Sigma \times \mathbb{C}$ lifts to $\mathcal{E}$. Indeed it is immediate to check that the action given by $\operatorname{diag}(t, 1, \mathrm{id}, t)$ in the local trivialization $\left.\mathcal{E}\right|_{\left(U_{i} \cap U_{j}\right) \times \mathbb{C}} \simeq \mathbb{C}^{\operatorname{rk}(F)} \oplus \mathbb{C}^{\mathrm{rk}(E / F)} \times$ $\left(U_{i} \cap U_{j}\right) \times \mathbb{C}$ commutes with $\tilde{e}_{i j}$. Thus projectivizing $\mathcal{E}$ and projecting to $\mathbb{C}$ gives a nonproduct test configuration $\mathbb{P}(\mathcal{E}) \rightarrow \mathbb{C}$ for $\mathbb{P}(E)$ with central fiber $\mathbb{P}(F \oplus E / F)$ acted on by $\mathbb{C}^{*}$ via $\operatorname{diag}(t, 1)$. Thanks to Proposition 3.1 the Chow weight of that configuration is nonnegative, whence it follows that $\mathbb{P}(E)$ is not polystable.

The slope stability of $E$ is related to the existence of $\operatorname{cscK}$ metrics on $\mathbb{P}(E)$ as stated in the following.

Theorem 3.6 (Apostolov, Calderbank, Gauduchon and Tønnesen-Friedman [1]). A projective bundle $\mathbb{P}(E)$ over a curve of genus $g \geq 2$ admits a cscK metric in some (and hence any) Kähler class if and only if $E$ is polystable.

Thus by Theorems 3.5 and 3.6 we get the following.

Theorem 3.7. A projective bundle $\mathbb{P}(E)$ over a curve of genus $g \geq 2$ is cscK if and only if it is asymptotically Chow polystable w.r.t. some (and hence any) polarization.

Theorem 3.7 above gives a new proof of the Yau-Tian-Donaldson conjecture for projective bundles once one shows that $K$-(poly)stability is equivalent to asymptotic Chow (poly)stability in this case. This is a subtle purely algebraic problem. On the other hand we notice that in this case the YTD conjecture follows by known results. Indeed by Ross and Thomas [25, Theorem 5.13] and Theorem [3.6, the K-polystability of $\mathbb{P}(E)$ implies the existence of a cscK metric. Conversely if $\mathbb{P}(E)$ admits a cscK metric, then it is $K$-polystable by Mabuchi 17, 19].

\section{Blowup OF POINTS}

Let $(M, L)$ be a polarized manifold of dimension $n \geq 2$. Let $S=\left\{p_{1}, \ldots, p_{s}\right\} \subset$ $M$ be a set of points and consider the blowup

$$
\beta: \tilde{M} \rightarrow M
$$

of $M$ at $S$ with exceptional divisors $E_{j}=\beta^{-1}\left(p_{j}\right)$. Let $m, \alpha_{1}, \ldots, \alpha_{s}$ be positive integers such that the line bundle $\tilde{L}=\beta^{*} L^{m} \otimes \mathcal{O}\left(-\sum_{j=1}^{s} \alpha_{j} E_{j}\right)$ is ample on $\tilde{M}$. Moreover suppose we are given a $\mathbb{C}^{*}$-action on $M$ that fixes every $p_{j}$. This induces an action on $\tilde{M}$ that makes $\beta$ an equivariant map, and it is known that any $\mathbb{C}^{*}$ action on $\tilde{M}$ has this form. Since each exceptional divisor $E_{j}$ is invariant, the 
associated line bundle $\mathcal{O}\left(-E_{j}\right)$ has a natural linearization, so that $\tilde{L}$ is a linearized line bundle. Finally let $\omega$ be an $S^{1}$-invariant Kähler form representing $c_{1}(L)$, and let $\phi$ be a moment map for $\omega$. The choice of $\phi$ is equivalent to fixing a linearization of the given action on $L$. Finally let $a_{\ell}(M, L)$ and $b_{\ell}(M, L)$ be defined as in (1) and (2).

Theorem 4.1. In the situation above, assume that $(M, L)$ is asymptotically Chow polystable. Let $\lambda\left(p_{j}\right)$ be the total weight of the isotropy action on $T_{p_{j}} M$. We have

$$
\operatorname{Chow}\left(\tilde{M}, \tilde{L}^{k}\right)=\frac{a_{0}\left(M, L^{m}\right)-\sum_{j=1}^{s} \alpha_{j}^{n} / n !}{\chi\left(M, L^{m k}\right)-\sum_{j=1}^{s}\left(\begin{array}{c}
n+\alpha_{j} k-1 \\
\alpha_{j} k-1
\end{array}\right)} \sum_{\ell=1}^{n} F_{\ell}(\tilde{M}, \tilde{L}) k^{n+1-\ell},
$$

where

$$
F_{\ell}(\tilde{M}, \tilde{L})=\frac{1}{D^{2} m^{\ell-1}} \sum_{j=1}^{s}\left(f_{\ell}\left(\frac{\alpha_{j}}{m}\right) \phi\left(p_{j}\right)-g_{\ell}\left(\frac{\alpha_{j}}{m}\right) \lambda\left(p_{j}\right)\right),
$$

$D=\operatorname{deg}(M, L)-\sum_{j=1}^{s}\left(\frac{\alpha_{j}}{m}\right)^{n}>0$, and $f_{\ell}, g_{\ell}$ are polynomial functions defined by

$$
\begin{gathered}
f_{\ell}(x)=D s_{n-\ell} x^{n-\ell}-\left(n ! a_{\ell}(M, L)-s_{n-\ell} \sum_{i=1}^{s}\left(\frac{\alpha_{i}}{m}\right)^{n-\ell}\right) x^{n}, \\
g_{\ell}(x)=\frac{D s_{n+1-\ell} x^{n+1-\ell}-x f_{\ell}(x)}{n+1}
\end{gathered}
$$

and $s_{h}$ are nonnegative rational numbers, depending on $n$, generated by $\left(\begin{array}{c}n+x-1 \\ x-1\end{array}\right)=$ $\sum_{h \geq 0} \frac{s_{h} x^{h}}{n !}$.

Proof. We start fixing some notation. Let $\mathcal{I}_{j} \subset \mathcal{O}_{M}$ be the ideal sheaf of the point $p_{j}$, and let $Z \subset M$ be the subscheme cut out by $\mathcal{I}_{Z}=\mathcal{I}_{1}^{\alpha_{1}} \cdots \mathcal{I}_{s}^{\alpha_{s}}$. For $k \gg 0$ we have $\beta_{*} \mathcal{O}\left(-k \sum_{j=1}^{s} \alpha_{j} E_{j}\right)=\mathcal{I}_{Z}^{k}$ and $R^{i} \beta_{*} \mathcal{O}\left(-k \sum_{j=1}^{s} \alpha_{j} E_{j}\right)=0$ for all $i>0$. Thus, by the projection formula we get $H^{0}\left(\tilde{M}, \tilde{L}^{k}\right) \simeq H^{0}\left(M, \mathcal{I}_{Z}^{k} \otimes L^{m k}\right)$ equivariantly and $H^{i}\left(M, \mathcal{I}_{Z}^{k} \otimes L^{m k}\right)=0$ for all $i>0$; hence we have to calculate the dimension and the weight of $H^{0}\left(M, \mathcal{I}_{Z}^{k} \otimes L^{m k}\right)$. To this end we consider the equivariant exact sequence

$$
0 \rightarrow \mathcal{I}_{Z}^{k} \otimes L^{m k} \rightarrow L^{m k} \rightarrow L^{m k} / \mathcal{I}_{Z}^{k} \otimes L^{m k} \rightarrow 0,
$$

and the induced sequence

$$
0 \rightarrow H^{0}\left(M, \mathcal{I}_{Z}^{k} \otimes L^{m k}\right) \rightarrow H^{0}\left(M, L^{m k}\right) \rightarrow H^{0}\left(M, L^{m k} / \mathcal{I}_{Z}^{k} \otimes L^{m k}\right) \rightarrow 0,
$$

which is equivariant and exact by the considerations above. The sheaf $L^{m k} / \mathcal{I}_{Z}^{k} \otimes$ $L^{m k}$ is supported on $S$, and we have the equivariant isomorphism

$$
H^{0}\left(M, L^{m k} / \mathcal{I}_{Z}^{k} \otimes L^{m k}\right) \simeq \bigoplus_{j=1}^{s} L_{p_{j}}^{m k} \otimes\left(\bigoplus_{d=0}^{\alpha_{j} k-1} \operatorname{Sym}^{d} T_{p_{j}}^{*} M\right),
$$


where $\mathbb{C}^{*}$ acts on $L_{p_{j}}$ with weight $-\phi\left(p_{j}\right)$ and on $T_{p_{j}}^{*} M$ with total weight $-\lambda\left(p_{j}\right)$. Thus, by easy calculations we get

$$
\chi\left(M, L^{m k} / \mathcal{I}_{Z}^{k} \otimes L^{m k}\right)=\sum_{j=1}^{s}\left(\begin{array}{c}
n+\alpha_{j} k-1 \\
\alpha_{j} k-1
\end{array}\right)
$$

and

$$
\begin{aligned}
w\left(M, L^{m k} / \mathcal{I}_{Z}^{k} \otimes L^{m k}\right) & \\
& =-\sum_{j=1}^{s}\left[\left(\begin{array}{c}
n+\alpha_{j} k-1 \\
\alpha_{j} k-1
\end{array}\right) m k \phi\left(p_{j}\right)+\left(\begin{array}{c}
n+\alpha_{j} k-1 \\
\alpha_{j} k-2
\end{array}\right) \lambda\left(p_{j}\right)\right] .
\end{aligned}
$$

Moreover, by (12) and the ampleness of $L$ and $\tilde{L}$ we obtain

$$
\begin{aligned}
\chi\left(\tilde{M}, \tilde{L}^{k}\right) & =\chi\left(M, L^{m k}\right)-\sum_{j=1}^{s}\left(\begin{array}{c}
n+\alpha_{j} k-1 \\
\alpha_{j} k-1
\end{array}\right) \\
& =\sum_{\ell=0}^{n} a_{\ell}(M, L)(m k)^{n-\ell}-\sum_{j=1}^{s} \sum_{h=0}^{n} \frac{s_{h}}{n !}\left(\alpha_{j} k\right)^{h} \\
& =\sum_{\ell=0}^{n}\left(a_{\ell}(M, L) m^{n-\ell}-\frac{s_{n-\ell}}{n !} \sum_{j=1}^{s} \alpha_{j}^{n-\ell}\right) k^{n-\ell} .
\end{aligned}
$$

Since $(M, L)$ is asymptotically Chow polystable, we can fix a linearization on $L$ such that $b_{\ell}(M, L)=0$ for all $\ell=0, \ldots, n$. Indeed, we can choose the moment map $\phi$ such that $b_{0}(M, L)=\int_{M} \phi \omega^{n} / n !=0$, and in this case the asymptotic Chow polystability implies $w\left(M, L^{k}\right)=0$ for all $k \gg 0$. Thus by (12) and (13) we have

$$
\begin{aligned}
& w\left(\tilde{M}, \tilde{L}^{k}\right)=\sum_{j=1}^{s}\left[\left(\begin{array}{c}
n+\alpha_{j} k-1 \\
\alpha_{j} k-1
\end{array}\right) m k \phi\left(p_{j}\right)+\left(\begin{array}{c}
n+\alpha_{j} k-1 \\
\alpha_{j} k-2
\end{array}\right) \lambda\left(p_{j}\right)\right] \\
& =\sum_{\ell=0}^{n}\left(\frac{s_{n-\ell}}{n !} m \sum_{j=1}^{s} \alpha_{j}^{n-\ell} \phi\left(p_{j}\right)+\frac{s_{n-\ell}-s_{n+1-\ell}}{(n+1) !} \sum_{j=1}^{s} \alpha_{j}^{n+1-\ell} \lambda\left(p_{j}\right)\right) k^{n+1-\ell} .
\end{aligned}
$$

Thanks to the smoothness of $\tilde{M}$, the first formula of the statement follows easily from the definition of $\operatorname{Chow}\left(\tilde{M}, \tilde{L}^{k}\right)$ and $F_{\ell}\left(\tilde{M}, \tilde{L}^{k}\right)$, and the identity

$$
\frac{a_{0}(\tilde{M}, \tilde{L})}{\chi\left(\tilde{M}, \tilde{L}^{k}\right)}=\frac{a_{0}\left(M, L^{m}\right)-\sum_{j=1}^{s} \alpha_{j}^{n} / n !}{\chi\left(M, L^{m k}\right)-\sum_{j=1}^{s}\left(\begin{array}{c}
n+\alpha_{j} k-1 \\
\alpha_{j} k-1
\end{array}\right)}
$$

which in turn is a straightforward consequence of equations (14), (15) and $s_{n}=1$. 
Moreover, in order to get formula (11), we calculate

$$
\begin{aligned}
F_{\ell}\left(\tilde{M}, \tilde{L}^{k}\right)= & \frac{a_{0}(\tilde{M}, \tilde{L}) b_{\ell}(\tilde{M}, \tilde{L})-a_{\ell}(\tilde{M}, \tilde{L}) b_{0}(\tilde{M}, \tilde{L})}{a_{0}(\tilde{M}, \tilde{L})^{2}} \\
= & \left(\frac{1}{\operatorname{deg}(M, L)-\sum_{j=1}^{s}\left(\frac{\alpha_{j}}{m}\right)^{n}}\right)^{2} \frac{a_{0}(\tilde{M}, \tilde{L}) b_{\ell}(\tilde{M}, \tilde{L})-a_{\ell}(\tilde{M}, \tilde{L}) b_{0}(\tilde{M}, \tilde{L})}{\left(m^{n} / n !\right)^{2}} \\
= & D^{-2}\left[( \operatorname { d e g } ( M , L ) - \sum _ { i = 1 } ^ { s } ( \frac { \alpha _ { i } } { m } ) ^ { n } ) \left(s_{n-\ell} \sum_{j=1}^{s}\left(\frac{\alpha_{j}}{m}\right)^{n-\ell} \phi\left(p_{j}\right)\right.\right. \\
& \left.+\frac{s_{n-\ell}-s_{n+1-\ell}}{n+1} \sum_{j=1}^{s}\left(\frac{\alpha_{j}}{m}\right)^{n+1-\ell} \lambda\left(p_{j}\right)\right) \\
& -\left(n ! a_{\ell}(M, L)-s_{n-\ell} \sum_{i=1}^{s}\left(\frac{\alpha_{i}}{m}\right)^{n-\ell}\right) \\
& \left.\times\left(\sum_{j=1}^{s}\left(\frac{\alpha_{j}}{m}\right)^{n} \phi\left(p_{j}\right)+\frac{1}{n+1} \sum_{j=1}^{s}\left(\frac{\alpha_{j}}{m}\right)^{n+1} \lambda\left(p_{j}\right)\right)\right] m^{1-\ell} \\
= & D^{-2}\left(\sum_{j=1}^{s} f_{\ell}\left(\frac{\alpha_{j}}{m}\right) \phi\left(p_{j}\right)-\sum_{j=1}^{s} g_{\ell}\left(\frac{\alpha_{j}}{m}\right) \lambda\left(p_{j}\right)\right) m^{1-\ell},
\end{aligned}
$$

whence (11) follows easily.

Remark 4.2. From formula (11) follows easily the asymptotic expansion of the invariants $F_{\ell}$ in the so-called "adiabatic limit", $m \rightarrow \infty$. In particular, for $\ell=1$ we have

$$
F_{1}(\tilde{M}, \tilde{L})=\frac{n(n-1)}{2 \operatorname{deg}(M, L)} \sum_{j=1}^{s}\left(\frac{\alpha_{j}}{m}\right)^{n-1} \phi\left(p_{j}\right)+O\left(\frac{1}{m^{n}}\right) .
$$

For each invariant point $p \in S$, the Chow weight of the zero-dimensional polarized scheme $\left(p, L_{p}\right)$ is clearly zero by definition. On the other hand, we define

$$
w_{\mathrm{CW}}(p, L)=\phi(p)-\frac{b_{0}(M, L)}{a_{0}(M, L)},
$$

and we extend by linearity to each zero-cycle $\sum_{j=1}^{s} c_{j} p_{j}$, with $c_{j} \in \mathbb{Q}$. Thus, recalling that $(M, L)$ is assumed to be asymptotically Chow polystable, we get

$$
F_{1}(\tilde{M}, \tilde{L})=\frac{n(n-1)}{2 \operatorname{deg}(M, L)} w_{\mathrm{C} W}\left(\sum_{j=1}^{s} \alpha_{j}^{n-1} p_{j}, L\right) \frac{1}{m^{n-1}}+O\left(\frac{1}{m^{n}}\right) .
$$

The same formula has been proved by Stoppa for a wider class of test configurations of blown-up manifolds [29].

Theorem 4.1 suggests that asymptotically Chow polystability is in general lost after blowing up if some nontrivial $\mathbb{C}^{*}$-action still remains. Indeed it is easy to find $\alpha_{1}, \ldots, \alpha_{s}$ and $m$ such that $F_{\ell}(\tilde{M}, \tilde{L}) \neq 0$, at least for some $\ell$. Refining this simple 
remark we can show by an example that blowing up a cscK asymptotically Chow polystable manifold can produce integral cscK classes which are asymptotically Chow unstable.

The strategy is the following. Given a Kähler manifold $M$, we know that the extremal cone is open in the Kähler cone by Lebrun and Simanca [15], and every extremal class with zero Futaki invariant is in fact cscK. Thus the set $\mathcal{C}$ of the cscK classes is open in the locus $\mathcal{F}$ where $F_{1}=0$. Moreover, when $M$ is a $\operatorname{cscK}$ manifold blown up at some points, $\mathcal{C}$ is nonempty under mild conditions by Arezzo and Pacard [3]. If we can prove that the locus $\mathcal{Z}$ where $F_{1}=\cdots=F_{n}=0$ is a proper Zariski closed subset of $\mathcal{F}$, and $\mathcal{C} \backslash \mathcal{Z}$ has at least a rational point, we are done. Clearly we look for a rational class in $\mathcal{C} \backslash \mathcal{Z}$ because it has to be (a rational multiple of) the first Chern class of a line bundle. In the example below we will show that rational points of $\mathcal{F}$ are in fact dense in the Euclidean topology.

Example 4.3. Let $(M, L)$ be the complex projective plane $\mathbb{P}^{2}$ polarized with the hyperplane bundle, and let $S=\left\{p_{1}, p_{2}, p_{3}, p_{4}\right\}$ be a set of points of $M$, and suppose that the $p_{j}$ 's are all but one aligned. Up to a projectivity we can suppose $p_{1}=$ $(1,0,0), p_{2}=(0,1,0), p_{3}=(0, a, b)$, and $p_{4}=(0, a,-b)$ with $a b \neq 0$. Let $(\tilde{M}, \tilde{L})$ be the blowup of $M$ along $S$ endowed with the polarization defined as in the general case above $\tilde{L}=\beta^{*} L^{m} \otimes \mathcal{O}\left(-\sum_{j=1}^{4} \alpha_{j} E_{j}\right)$.

The unique (up to scaling) holomorphic vector field of $\tilde{M}$ generates the $\mathbb{C}^{*}$ action $\operatorname{diag}\left(t^{2}, t^{-1}, t^{-1}\right)$ on $\mathbb{P}^{2}$; thus the condition $F_{\ell}(\tilde{M}, \tilde{L})=0$ in the Kähler cone is equivalent to a (homogeneous polynomial) equation in the variables $(m, \alpha)$.

By direct calculations from (11) we get $F_{1}(\tilde{M}, \tilde{L})=\operatorname{deg}(\tilde{M}, \tilde{L})^{-2} \psi_{1}(m, \alpha)$, where

(17) $\quad \psi_{1}(m, \alpha)=\left(2 \alpha_{1}-\alpha_{2}-\alpha_{3}-\alpha_{4}\right)\left(m^{3}-3 \alpha_{1}^{2} m\right)$

$-\left(2 \alpha_{1}^{2}-\alpha_{2}^{2}-\alpha_{3}^{2}-\alpha_{4}^{2}\right)\left(3 m^{2}-3 \alpha_{1} m\right)+\left(2 \alpha_{1}^{3}-\alpha_{2}^{3}-\alpha_{3}^{3}-\alpha_{4}^{3}\right)\left(3 m-\alpha_{1}-\alpha_{2}-\alpha_{3}-\alpha_{4}\right)$,

and $F_{2}(\tilde{M}, \tilde{L})=\operatorname{deg}(\tilde{M}, \tilde{L})^{-2} \psi_{2}(m, \alpha)$, where

$$
\begin{gathered}
\psi_{2}(m, \alpha)=\left(2 \alpha_{1}-\alpha_{2}-\alpha_{3}-\alpha_{4}\right)\left(m^{2}-\alpha_{1}^{2}-\alpha_{2}^{2}-\alpha_{3}^{2}-\alpha_{4}^{2}\right) \\
-2\left(2 \alpha_{1}^{2}-\alpha_{2}^{2}-\alpha_{3}^{2}-\alpha_{4}^{2}\right) m+2\left(2 \alpha_{1}^{3}-\alpha_{2}^{3}-\alpha_{3}^{3}-\alpha_{4}^{3}\right) .
\end{gathered}
$$

Since $\psi_{1}$ and $\psi_{2}$ are irreducible and in particular $\psi_{2}$ does not divide $\psi_{1}$, the locus $\psi_{2} \neq 0$ is nonempty and Zariski open in the locus $\psi_{1}=0$. On the other hand, it is easy to see that $(1,1,0,0,0) \in \mathbb{P}^{4}$ is a triple point for the quartic threefold defined by $\psi_{1}=0$; thus the set of rational solutions of the equation $\psi_{1}(m, \alpha)=0$ is dense in the Euclidean topology in the locus $\psi_{1}=0$. Since the latter is nonempty by Arezzo and Pacard [3, Example 7.3], we get that there are infinitely many integral cscK classes on $\tilde{M}$ which are asymptotically Chow unstable.

Example 4.4. The argument above shows that all rational cscK classes on the blowup of $\mathbb{P}^{2}$ along a set $S=\left\{p_{1}, p_{2}, p_{3}\right\}$ of three nonaligned points are asymptotically Chow stable. Indeed in this case the null loci of $F_{1}$ and $F_{2}$ are the same.

More precisely, assume without loss of generality that $p_{1}=(1,0,0), p_{2}=(0,1,0)$, $p_{3}=(0,0,1)$ and let $(\tilde{M}, \tilde{L})$ be the blowup of $\mathbb{P}^{2}$ along $S$ endowed with the polarization $\tilde{L}$ defined as above. A set of generators for the space of holomorphic vector fields of $\tilde{M}$ is now given by the fields associated with the $\mathbb{C}^{*}$-actions $\operatorname{diag}\left(t, t^{-1}, 1\right)$ and $\operatorname{diag}\left(1, t, t^{-1}\right)$ on $\mathbb{P}^{2}$. After a straight calculation involving formula (11), we see 
that $F_{1}(\tilde{M}, \tilde{L})=0$ if and only if

$\left\{\left(\alpha_{1}-\alpha_{2}\right)\left(m-\alpha_{1}-\alpha_{2}-\alpha_{3}\right)\left(m^{2}-2 \alpha_{1} m-2 \alpha_{2} m+\alpha_{3} m+\alpha_{1}^{2}+\alpha_{1} \alpha_{2}+\alpha_{2}^{2}\right)=0\right.$

$\left\{\left(\alpha_{2}-\alpha_{3}\right)\left(m-\alpha_{1}-\alpha_{2}-\alpha_{3}\right)\left(m^{2}-2 \alpha_{3} m-2 \alpha_{2} m+\alpha_{1} m+\alpha_{3}^{2}+\alpha_{3} \alpha_{2}+\alpha_{2}^{2}\right)=0\right.$

while $F_{2}(\tilde{M}, \tilde{L})=0$ is equivalent to

$$
\left\{\begin{array}{l}
\left(\alpha_{1}-\alpha_{2}\right)\left(m-\alpha_{1}-\alpha_{2}-\alpha_{3}\right)\left(m-\alpha_{1}-\alpha_{2}+\alpha_{3}\right)=0 \\
\left(\alpha_{2}-\alpha_{3}\right)\left(m-\alpha_{1}-\alpha_{2}-\alpha_{3}\right)\left(m+\alpha_{1}-\alpha_{2}-\alpha_{3}\right)=0 .
\end{array}\right.
$$

In order for $L_{m}$ to be ample, by Nakai's criterion, it must be that $m>0, \alpha_{i}>0$, and $m+\alpha_{j}>\alpha_{1}+\alpha_{2}+\alpha_{3}$ for all $j=1,2,3$; thus one immediately sees that $F_{1}(\tilde{M}, \tilde{L})=0$ if and only if $F_{2}(\tilde{M}, \tilde{L})=0$, and both are zero if and only if either $\alpha_{1}=\alpha_{2}=\alpha_{3}$ or $\alpha_{1}+\alpha_{2}+\alpha_{3}=m$ (compare with [15, Example 3.2]).

\section{ACKNOWLEDGMENTS}

The first named author would like to thank G. La Nave and G. Tian for stimulating discussions. Both authors would like to thank C. Arezzo for the encouragement and the interest in this work. The research of the first named author is supported by a Marie Curie International Outgoing Fellowship within the $7^{\text {th }}$ European Community Framework Programme (program CAMEGEST, proposal no. 255579).

\section{REFERENCES}

[1] V. Apostolov, D. M. J. Calderbank, P. Gauduchon and C. W. Tønnesen-Friedman, Extremal Kähler metrics on projective bundles over a curve. arxiv:0905.0498v1 [math.DG].

[2] C. Arezzo and F. Pacard, Blowing up and desingularizing constant scalar curvature Kähler manifolds. Acta Math. 196 (2006), no. 2, 179-228. MR2275832 (2007i:32018)

[3] C. Arezzo and F. Pacard, Blowing up Kähler manifolds with constant scalar curvature, II. Ann. of Math. (2) $\mathbf{1 7 0}$ (2009), no. 2, 685-738. MR2552105 (2010m:32025)

[4] M. F. Atiyah and R. Bott, The moment map and equivariant cohomology. Topology 23 (1984), no. 1, 1-28. MR.721448 (85e:58041)

[5] N. Berline, E. Getzler and M. Vergne, Heat kernels and Dirac operators. Springer-Verlag, 2004. MR.2273508 (2007m:58033)

[6] S. K. Donaldson, Scalar curvature and projective embeddings I. J. Differential Geom. 59 (2001), no. 3, 479-522. MR1916953 (2003j:32030)

[7] S. K. Donaldson, Scalar curvature and stability of toric varieties. J. Differential Geom. 59 (2002), no. 2, 289-349. MR 1988506 (2005c:32028)

[8] S. K. Donaldson, Constant scalar curvature metrics on toric surfaces. Geom. Funct. Anal. 19 (2009), no. 1, 83-136. MR2507220(2010j:32041)

[9] A. Futaki, Asymptotic Chow semi-stability and integral invariants. Internat. J. Math. 15, no. 9 (2004), 967-979. MR2106156 (2005g:32029)

[10] A. Futaki, Kähler-Einstein metrics and Integral Invariants. Lecture Notes in Mathematics 1314. Springer-Verlag, 1988. MR947341 (90a:53053)

[11] A. Futaki, H. Ono and Y. Sano, Hilbert series and obstructions to asymptotic semistability. Adv. Math. 226 (2011), 254-284. MR2735758

[12] A. Grothendieck, Éléments de géométrie algébrique III, part 2, Inst. Hautes Études Sci. Publ. Math. No. 17, 1963. MR0163911(29:1210)

[13] R. Hartshorne, Algebraic Geometry. Springer, 1977. MR0463157 (57:3116)

[14] F. F. Knudsen and D. Mumford, The projectivity of the moduli space of stable curves I: Preliminaries on "det" and "Div". Math. Scand. 39 (1976), no. 1. MR0437541 (55:10465)

[15] C. Lebrun and S. R. Simanca, Extremal Kähler metrics and complex deformation theory. Geom. Funct. Anal. 4 (1994), no. 3, 298-336. MR1274118 (95k:58041)

[16] T. Mabuchi, An energy-theoretic approach to the Hitchin-Kobayashi correspondence for manifolds. I. Invent. Math. 159 (2005), no. 2, 225-243. MR.2116275 (2005m:32047)

[17] T. Mabuchi, K-stability of constant scalar curvature polarization. arXiv:0812.4093v2 [math.DG]. 
[18] T. Mabuchi, Chow-stability and Hilbert-stability in Mumford's Geometric Invariant Theory. Osaka J. Math. 45 (2008), 833-846. MR2468597(2010b:14091)

[19] T. Mabuchi, A stronger concept of K-stability. arXiv:0910.4617v1 [math.DG].

[20] I. Morrison, Projective stability of ruled surfaces. Inventiones Math. 56 (1980), 269-304. MR.561975 (81c:14007)

[21] D. Mumford, Stability of projective varieties. L'enseignement mathématique XXIII (1977), no. 1-2, 39-110. MR0450272 (56:8568)

[22] M. S. Narasimhan and C. S. Seshadri, Stable and unitary vector bundles on a compact Riemann surface. Ann. of Math. (2) 82 (1965), no.3, 540-567. MR0184252 (32:1725)

[23] H. Ono, Y. Sano and N. Yotsutani, An example of asymptotically Chow unstable manifolds with constant scalar curvature. arXiv:0906.3836v1.

[24] S. T. Paul and G. Tian, CM stability and the generalized Futaki invariant I. arXiv:math/ 0605278v5 [math.AG].

[25] J. Ross and R. Thomas, An obstruction to the existence of constant scalar curvature Kähler metrics. J. Differential Geom. 72 (2006), no. 3, 429-466. MR2219940 (2007c:32028)

[26] J. Ross and R. Thomas, A study of the Hilbert-Mumford criterion for the stability of projective varieties. J. Algebraic Geom. 16 (2007), no. 2, 201-255. MR2274514 (2007k:14091)

[27] R. Seyyedali, Balanced metrics and Chow stability of projective bundles over Kähler manifolds. Duke Math. J. 153 (2010), no. 3, 573-605. MR2667426

[28] J. Stoppa, K-stability of constant scalar curvature Kähler manifolds. Adv. Math. 221 (2009), no. 4, 1397-1408. MR2518643 (2010d:32024)

[29] J. Stoppa, Unstable blowups. J. Algebraic Geom., 19 (2010), no. 1, 1-17. MR2551756 (2011c:32042)

[30] G. Tian, Kähler-Einstein metrics with positive scalar curvature. Invent. Math. 130 (1997), no. 1, 1-37. MR.1471884 (99e:53065)

[31] G. Tian, Extremal metrics and geometric stability. Houston Math. J. 28 (2002), no. 1, 411432. MR.1898198 (2003i:53062)

[32] S.-Y. Yau, Open problems in Geometry, in 'Differential geometry: partial differential equations on manifolds' (Los Angeles, CA, 1990), Proc. Sympos. Pure Math. 54 (1993), part 1, 1-28. MR1216573 (94k:53001)

[33] S. Zhang, Heights and reductions of semi-stable varieties. Compositio Math. 104 (1996), no. 1, 77-105. MR1420712 (97m:14027)

Department of Mathematics, Fine Hall, Princeton University, Princeton, New JerSey 08544 - And - Dipartimento di Matematica, Università degli Studi di Parma, Viale G. P. Usberti, 53/A, 43100 Parma Italy

E-mail address: della@math.princeton.edu

Dipartimento di Matematica, Università degli Studi di Parma, Viale G. P. Usberti, 53/A, 43100 PARMa ITALY

E-mail address: fabio.zuddas@unipr.it 